\title{
Modified Abbé flap for reconstruction of Cupid's bow and vermilion tubercle in secondary cleft lip deformity
}

\author{
Jun Won Lee, \\ Seong Joo Lee, \\ In Suck Suh, \\ Chong Kun Lee \\ Department of Plastic and Reconstructive \\ Surgery, Kangnam Sacred Heart Hospital, \\ Hallym University College of Medicine, Seoul, \\ Korea
}

\begin{abstract}
Background: Abbé flap technique is one of the most challenging operations to correct horizontal deficiencies in secondary cleft lip deformity. Since its first introduction, the operative method was dynamically modified from simple variation to complete conceptual change, but conventional Abbé flap has many drawbacks in esthetic and functional aspect. Our purpose was reconstructing the symmetry of Cupid's bow and central vermilion tubercle with minimal sequalae.

Methods: From 2008 to 2016 , this technique was applied to 16 secondary cleft lip patients who had total or more than $60 \%$ of unilateral deficiency of Cupid's bow and central lip or tubercle pouting deficiency. A quadrangular-shaped flap was transferred from vermilion including skin and white line of central or contralateral lower lip. Pedicle division and insetting were made at 9 (unilateral) or 10 (bilateral) days after transfer. Secondary lip revision was done with open rhinoplasty after wound maturation.

Results: Overall satisfaction was high with modified technique. Scar was minimally noticeable on both upper and lower lip especially. Balanced Cupid's bow and symmetric vermilion tubercle were made with relatively small size of flap compared to conventional Abbé flap. An accompanying benefit was reduced ectropion of lower lip, which made balanced upper and lower lip protrusion with more favorable profile.

Conclusion: A new modified Abbé flap technique showed great satisfaction. It is worth considering in secondary cleft lip patient who has central lip shortage and asymmetry of upper lip vermilion border line. Our technique is one of the substitutes for correction of horizontal and central lip deficiency with asymmetric Cupid's bow.
\end{abstract}

Keywords: Cleft lip / Surgical flaps / Esthetics

\section{INTRODUCTION}

In secondary cleft lip deformities, tight upper lip, absent or asymmetric Cupid's bow, central lip and tubercle pouting deficiency are not treated efficiently with simple revision surgery $[1]$. The $\mathrm{Cu}-$ pid' bow and central tubercle are a cardinal structures for sym-

\section{Correspondence: Chong Kun Lee}

Department of Plastic and Reconstructive Surgery, Kangnam Sacred Heart Hospital, Hallym University College of Medicine, 1 Singil-ro, Yeongdeungpo-gu, Seoul 07441, Korea E-mail: chong5184@hanmail.net

*This article was presented as a poster at the PRS KOREA 2016, the 74th Congress of the Korean Society of Plastic and Reconstructive Surgeons, Seoul, Korea

Received December 9, 2017 / Revised February 23, 2018 / Accepted March 8, 2018 metric upper lip [2]. They do not provide simple solution for lack of upper lip tissue and structural restoration. The concept of brining lower lip tissue into insufficient upper lip has long been introduced almost 200 years before, which was described by Robert Abbé, so-called "lip switch flap" in 1898 [3]. This technique became one of the most challenging operations in secondary cleft lip surgery. Conventional Abbé flap requires long incision and bulky tissue, which led to many drawbacks such as long and newly additional scar on both upper and lower lips [4]. A long while, many authors have contemplated to modify the Abbé flap to improve the results [5-9]. We would like to introduce a novel method 
to reconstruct the symmetry of Cupid's bow and central tubercle with minimal sequalae.

\section{METHODS}

From 2008 to 2016, this technique was applied to 16 patients who had total or more than $60 \%$ of unilateral deficiency of Cupid's bow and central lip or tubercle pouting deficiency in those of patients with secondary cleft lip deformities. The surgeries were conducted by single surgeon (C.K.L.). The basic demographics such as diagnosis, age, sex, short of Cupid's bow, and flap size are documented. Preoperative measuring included the length and peak-point level of Cupid's bow in normal and cleft side. The shortage of length and size of the transferring tissue was carefully contemplated and documented as well (Tables 1,2). The medical records and patient response were investigated. The operator and another plastic surgeon were participated in evaluation of outcome by any methods such as direct inspection, subjective satisfaction and clinical photographs, using visual analogue scale (VAS) graded from 0 (worst outcome) to 10 (best outcome) before and after the surgery. Interobserver consistency was measured with Cronbach's $\alpha$ and analyzed with IBM SPSS ver. 22.0 (IBM Corp., Armonk, NY, US). Statistical significance was defined when $p$-value was less than 0.05 .

\section{Operative technique}

\section{Unilateral cleft lip deformity}

Under either general or local anesthesia, the patient was supine position with mild neck extension. The full face was prepared in usual sterile manner. Operative design was made differently in terms of shape and location according to the diagnosis of the patient, whether it was bilateral or unilateral cases (Fig. 1). The local injection was made with $1 \%$ lidocaine and 1:200,000 epinephrine solutions at the recipient and donor site. The eccentric previous surgical scar was resected to keep up the symmetric slant with normal vermilion border line as same manner (Fig. 1B, C). The defect was widened by splitting and meticulous trimming of scar tissue inch by inch even though normal tissue, which released

Table 1. Patient demographics of unilateral cleft lip

\begin{tabular}{ccccccc} 
Patient no. & Age $(\mathrm{yr})$ & Sex & Diagnosis & L1 $(\mathrm{mm})$ & L2 $(\mathrm{mm})$ & Flap size $(\mathrm{mm})$ \\
\hline 1 & 13 & Male & Unilateral cleft lip & 5.0 & 1.0 & 8.0 \\
4 & 25 & Male & Unilateral cleft lip & 7.9 & 1.0 & 7.0 \\
\hline 5 & 33 & Male & Unilateral cleft lip & 7.5 & 7.4 & 7.0 \\
7 & 29 & Male & Unilateral cleft lip & 7.9 & 2.0 & 7.0 \\
\hline 8 & 22 & Male & Unilateral cleft lip & 8.9 & 8.0 & 7.5 \\
9 & 37 & Male & Unilateral cleft lip & 8.7 & 1.0 & 7.0 \\
\hline 10 & 50 & Female & Unilateral cleft lip & 8.0 & 0.0 & 8.0 \\
\hline
\end{tabular}

L1, length of Cupid's bow on normal side; L2, remained length of Cupid's bow on cleft side.

Table 2. Patient demographics of bilateral cleft lip

\begin{tabular}{|c|c|c|c|c|c|}
\hline Patient no. & Age (yr) & Sex & Diagnosis & Clinical feature & Flap size (mm) \\
\hline 2 & 27 & Male & Bilateral cleft lip & Absence of Cupid's bow and central deficiency & 9.0 \\
\hline 3 & 32 & Male & Bilateral cleft lip & Absence of Cupid's bow and central deficiency & 9.8 \\
\hline 6 & 31 & Male & Bilateral cleft lip & Absence of Cupid's bow and central deficiency & 9.0 \\
\hline 11 & 20 & Female & Bilateral cleft lip & Central deficiency & 7.5 \\
\hline 12 & 13 & Male & Bilateral cleft lip & Scar of Cupid's bow and central deficiency & 7.0 \\
\hline 13 & 18 & Male & Bilateral cleft lip & Central deficiency & 9.0 \\
\hline 14 & 21 & Male & Bilateral cleft lip & Scar of Cupid's bow and central deficiency & 9.0 \\
\hline 15 & 35 & Female & Bilateral cleft lip & Central deficiency & 10 \\
\hline 16 & 18 & Male & Bilateral cleft lip & Central deficiency & 10 \\
\hline
\end{tabular}



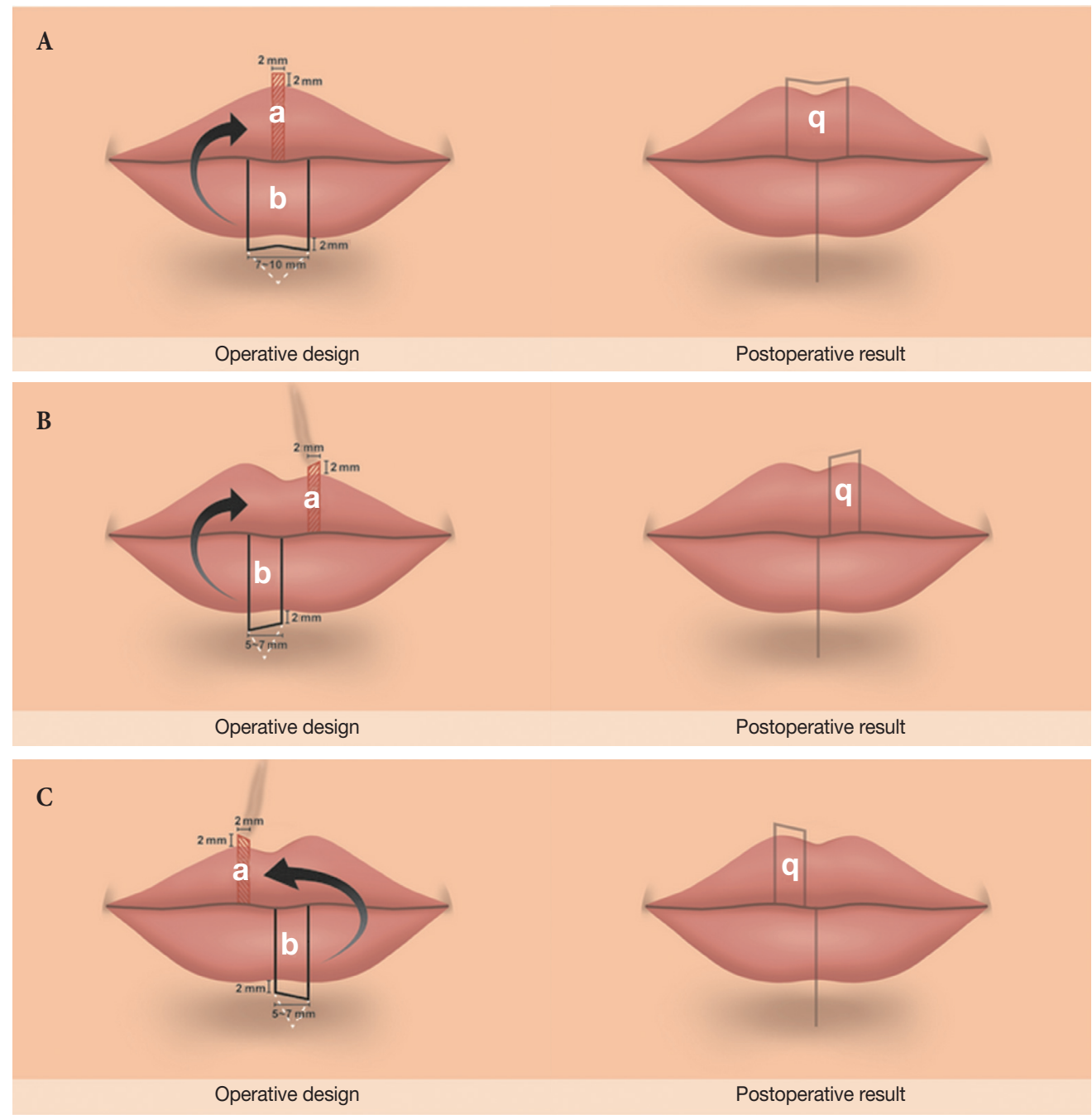

Fig. 1. Overall design of the modified Abbé flap for Cupid's bow reconstruction in bilateral cleft lip deformity (A), left side (B) and right side of lip (C) in unilateral cleft lip deformity. Scar tissue to be excised and released (a), flap to be transferred and inset (b) and area to be discarded or deepithelialized, for primary closure of the donor site (white-dotted area).

from tightness of upper lip partially. The proper recipient space was made as equal size of flap which being a same length of normal vermilion border line including remaining length.

A pentagonal-shaped flap was designed as 6 to $10 \mathrm{~mm}$-wide vermilion including $2 \mathrm{~mm}$-high skin and white line from central or contralateral side of lower lip (Fig. 1). Donor site was closed primarily, in which the scar was not over the labiomental sulcus. After confirmation of intact blood flow, the medial-based labial artery pedicled flap was transferred to clockwise or counterclockwise fashion to recipient site. The triangular part of the distal tip of the flap was discarded or deepithelialized and inserted into ready-made space of upper lip. The flap was closed layer by layer in order of mucosa, muscle, subcuticular layer and skin (Fig. 2A, B). Pedicle division and flap insetting were made 9 days after the initial surgery under local anesthesia after conformation flap viability by pedicle ligation for 30 minutes. Further lip revision combined with open rhinoplasty was done after wound maturation for more aesthetic outcome.

\section{Bilateral cleft lip deformity}

The overall procedures were similar to the operation in unilateral cleft lip patient. The difference was the location and size of flap 

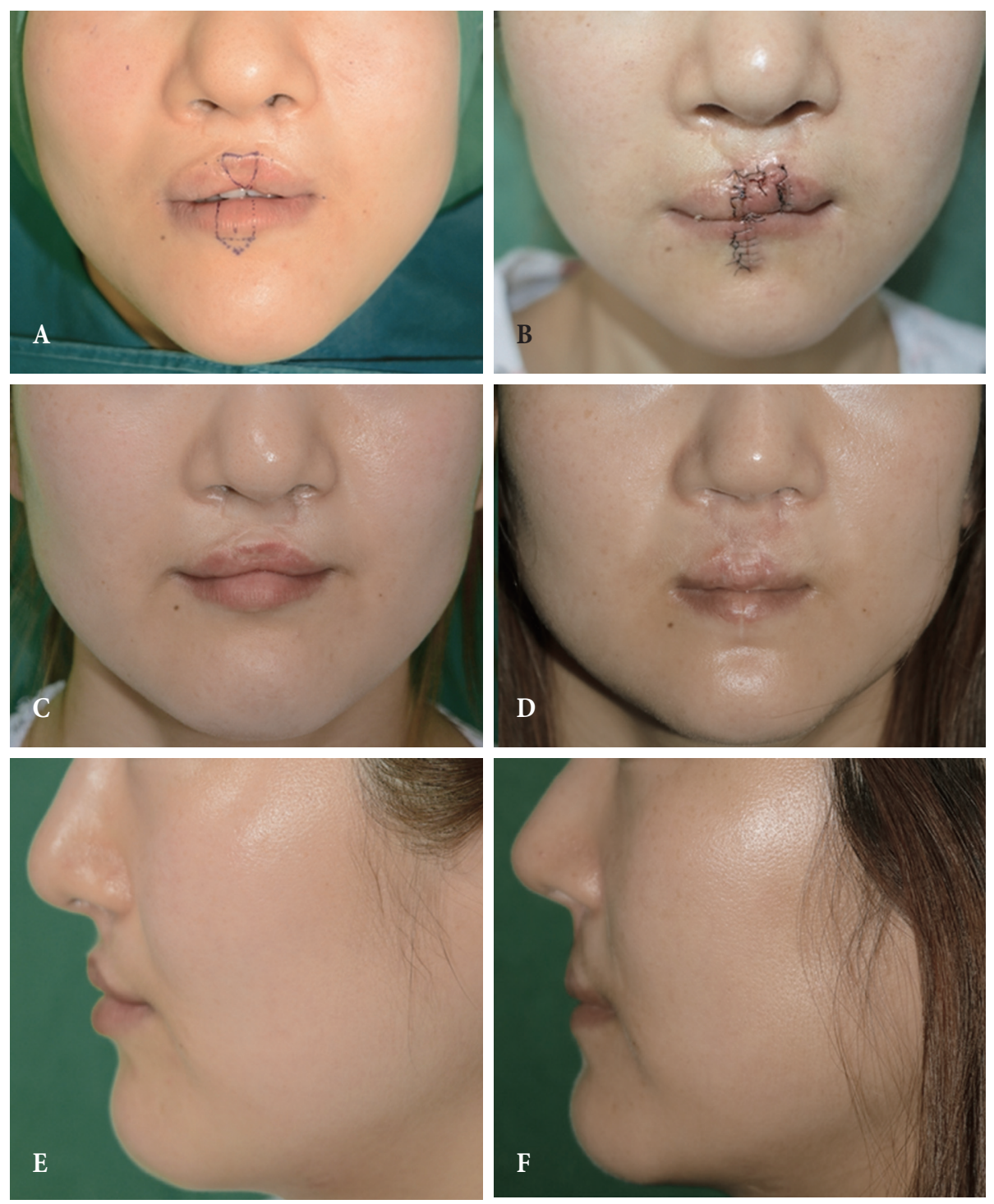

Fig. 2. Intraoperative clinical photographs of Abbé flap design with flap transfer (A, B; case 15), before Abbé flap and 2 years after the flap transfer including open rhinoplasty and two stages of scar revision (C, D), and preoperative and postoperative appearance ( 2 years) of lip protrusion and profile (E, F).

which was from center of lower lip from 7 to $10 \mathrm{~mm}$ in width (Fig. 1A). The recipient site was prepared by excision of tiny tissue including midline notching scar. The gap was enlarged by splitting and meticulous trimming to make same space with flap size. If the Cupid's bow was normal ranged state, the disfigured and notched vermilion mucosa was excised only. Then, the flap was transferred from mucosal part of lower lip vermilion without white line and skin. Pedicle division and flap insetting were made 10 days after the initial surgery.

\section{RESULTS}

Mean patient age was 26.5 years (range, 13 to 50 years); 13 were males and three were females. There were nine bilateral cleft lip and seven unilateral cleft lip patients. Overall satisfaction was high with modified Abbé flap operation in terms of shape and symmetry. Scar was barely noticeable. However, the color of vermilion mucosa takes a long time to similar with on site color of upper lip mucosa. Most of the patient showed balanced Cupid's bow and 
relatively symmetric vermilion border line. Some patients were revised of scar and convolutional vermilion line. An accompanying benefit was reduced ectropion of lower lip, which made balanced upper and lower lip protrusion and favorable profile (Figs. 2-5). There was no acute complication regarding operation site. The median VAS score was 4 (range, 3 to 5) in bilateral cleft lip patients and 5 (range, 3 to 6) in unilateral cleft lip patients preoperatively, and 7 (range, 5 to 8 ) in bilateral cleft lip patients and 6 (range, 5 to 8) in unilateral cleft lip patients postoperatively from one observer. The other observer found the same results, while the median VAS score was 7 (range, 5 to 8 ) in bilateral cleft lip patients. Interobserver consistency was high in both preoperative and postoperative evaluation (Cronbach's $\alpha=0.831, p=0.001$ preoperatively and Cronbach's $\alpha=0.803, p=0.002$ postoperatively).

\section{DISCUSSION}

Abbé flap technique was modified dynamically since its first introduction in 1898 [3]. Our technique is unique in its conception, design and flap size. Minimal lip tissue is transferred for less mor- bidity and minimal scar, but a small part of vermilion border line and skin is essential for making Cupid's bow which is disfigured or absent in almost all secondary cleft lip deformity patients. The lower lip is the only substitutive source for upper lip which severely disfigured vermillion border line or short of Cupid's bow. Unilateral cleft lip deformity has short of Cupid's bow in cleft side over 90\% of patient in our experience. The flap must be transferred from contralateral side of lower lip for restoration of vermilion border line gradient. In bilateral cleft lip deformity, the Cupid's bow was substituted totally for symmetry. The middle segment of lower lip presents an excellent substitute for Cupid's bow. In unilateral cleft lip which has normal Cupid's bow in one side and abnormal one in cleft side, the flap can be transferred from contralateral side to restore volume and symmetry in upper lip.

The lower lip asymmetry is not as much noticeable as in upper lip, if it is not over $7 \mathrm{~mm}$ wide of flap size in unilateral cleft lip deformity. The flap width should not exceed $10 \mathrm{~mm}$ wide to prevent eminent deformity and unnatural movability of lower lip so far as in bilateral cleft lip deformity. Although some studies suggest 10 to $15-\mathrm{mm}$ width for optimal flap size, the characteristics of the
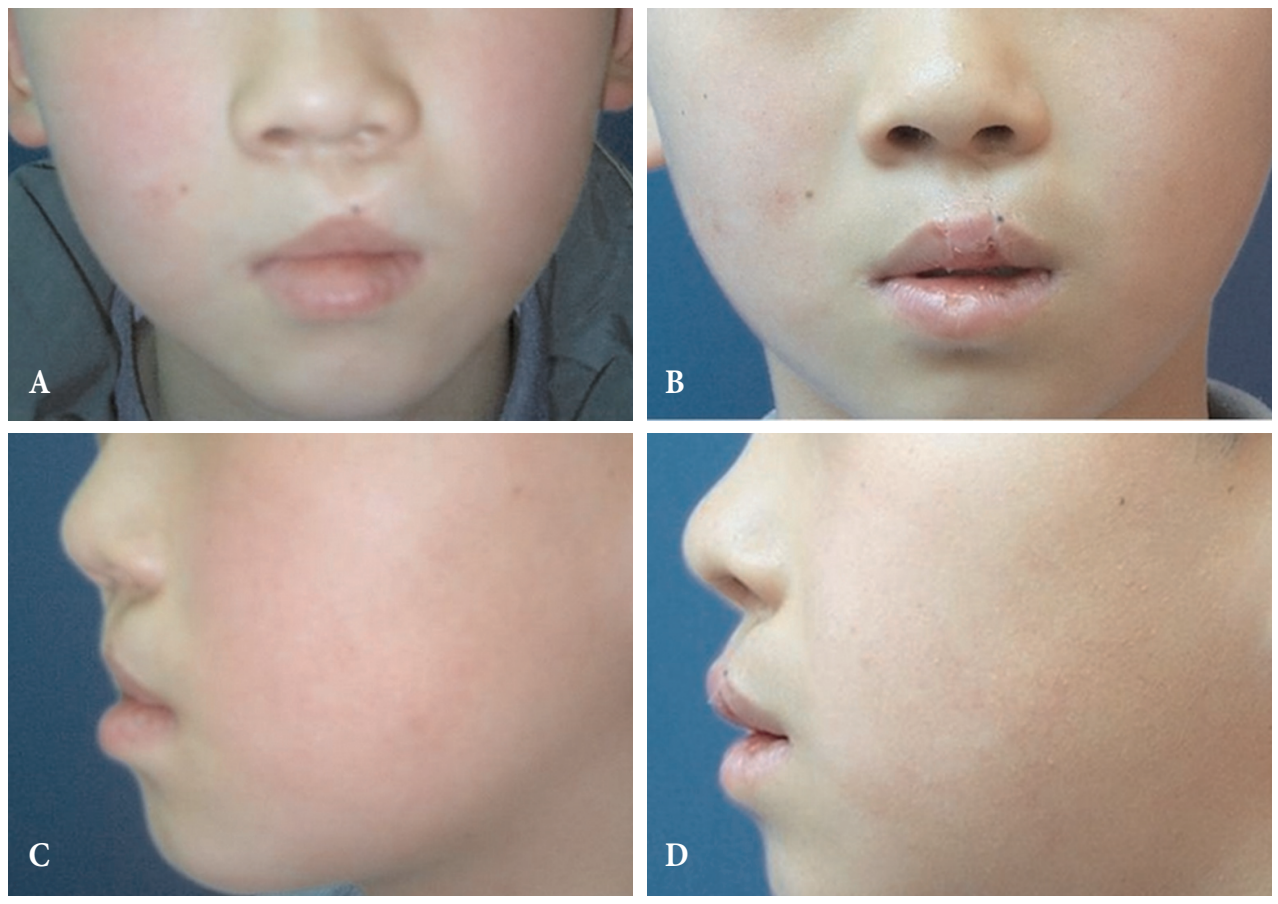

Fig. 3. Preoperative (A) and postoperative (B) clinical photos of Cupid's bow reconstruction (case 1) and lip protrusion and profile (C, D). Postoperative photos were taken just before any revisions, 6 months after Abbé flap. 

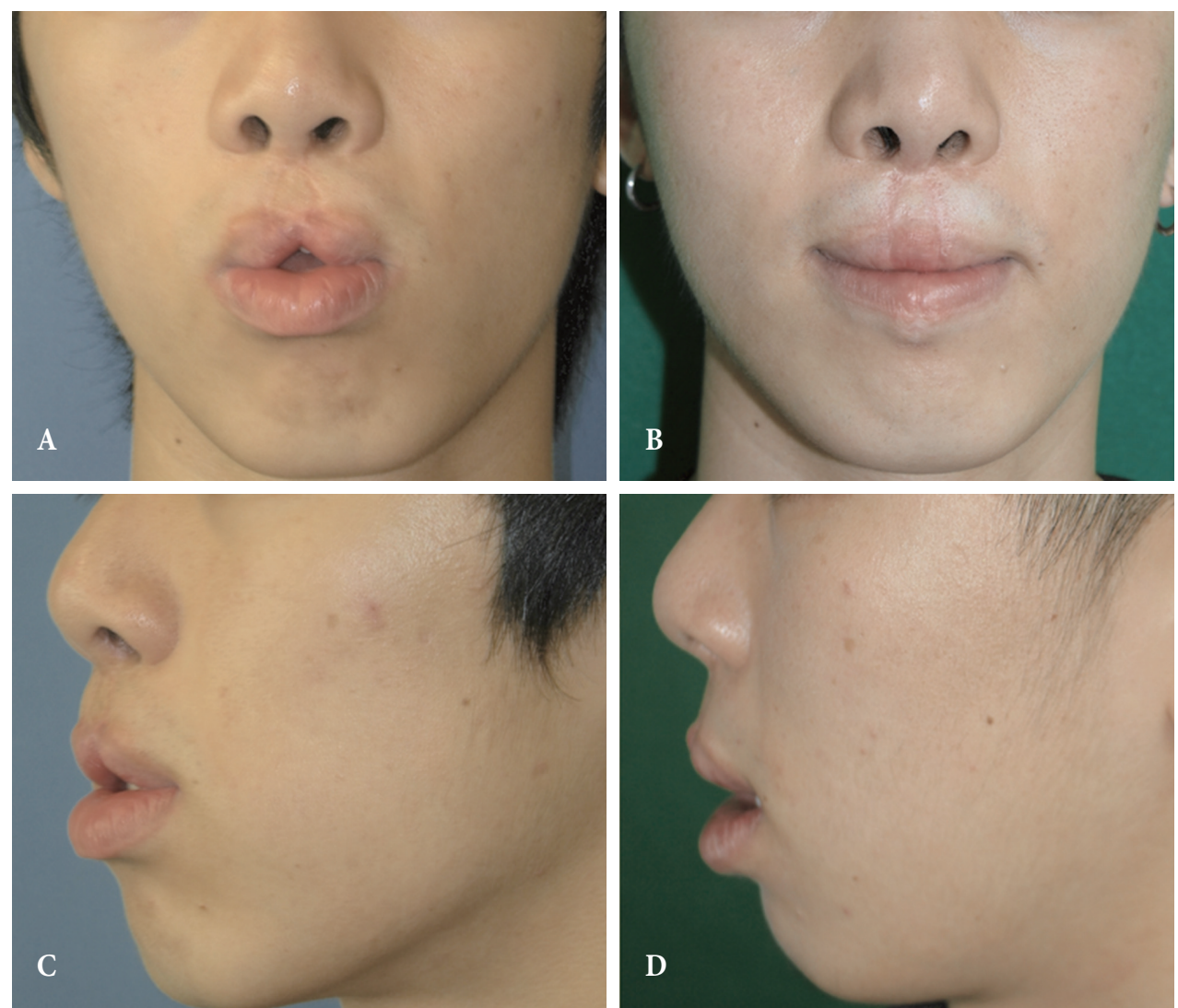

Fig. 4. Preoperative (A) and postoperative (4 years, B) clinical photos of Cupid's bow reconstruction (case 14) and lip protrusion and profile (C, D). The patient underwent lip reduction, open rhinoplasty, and four rounds of laser treatment.
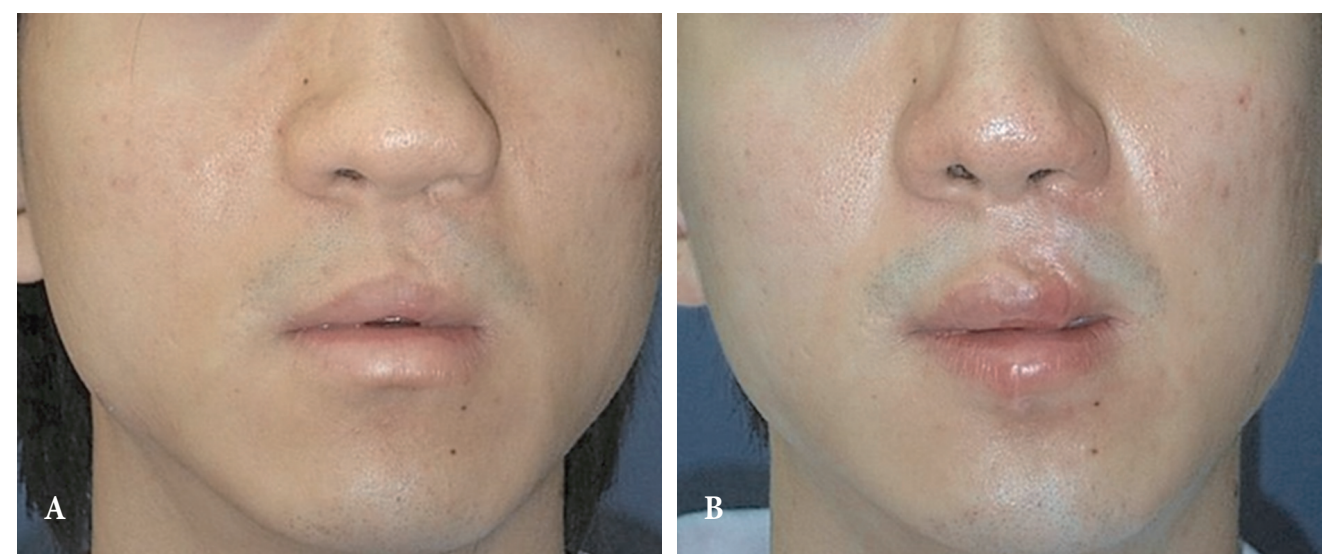

Fig. 5. Preoperative (A) and postoperative (B) clinical photos of left Cupid's bow reconstruction (case 3). Postoperative photos were taken just before any revisions, 6 months after Abbé flap.

upper and lower lips of each patient were taken into account to determine the appropriate size [10]. The lengths of the upper and lower lips in each patient were measured before entering and in the operating room, and the flap size was decided based on the subtracted value of the two lengths.

Pedicle was divided 9 days after the surgery in the unilateral cleft 
lip patients, and 10 days in the bilateral cleft lip patients. A report suggest even waiting only 5 days before severing the pedicle is safe enough [11]. However, there was concern of patients' movement of the attached lips, compromising the vascular supply, since the patients were discharged after the initial surgery. Furthermore, the pedicle was not divided before testing of the flap viability via ligation. Cleft lip patients usually require further scar revisions of the resulting upper lip scar from previous surgeries, and upper lip scars resulting from Abbé flap can be corrected simultaneously. Thus, the scar from delaying flap division and insetting was not such a big concern. The safety of the flap was on the highest priority.

One usual complaint of patients with Abbé flap is new and additional scar on upper and lower lip. In conventional technique or other modifications, long scar of lower lip and multiple linear scars on upper lip were inevitable. In this technique, lower lip scar is limited to labiomental sulcus and shaded area which scar can hide even in frontal view. In terms of upper lip, no new scar is formed on philtral area except small transverse short scar whose height is less than $2 \mathrm{~mm}$. Furthermore, in unilateral cleft lip patients, no scar is formed even in the upper lip mucosa by using existing scar from primary repair. This is the major advantage from other techniques, which leave long and highly-visible scar on upper and lower lip, occasionally extending to nostril sill. Minimal intervention induced the best result on esthetic and functional aspect on both upper and lower lip.

Small amount of tissue dissection and flap harvesting contribute to excellent versatility of the flap. No complication was shown regarding flap loss or even marginal necrosis. The flap color and volume showed disharmonious with adjacent vermilion mucosa which was turned into similarity over time. Some patients had notching of insertion site on mucosa, which were resolved with simple scar revision.

One additional benefit of this technique is improved of upper and lower lip relationship in lateral profile. Almost secondary cleft lip patients usually have unfavorable profile resulting from retrusion of upper lip and greater volume and eversion of lower lip especially in mandibular prognathism. This makes the lower lip protrusion and ectropion with opening mouth. This technique makes the lower lip tightening and closing mouth with more harmonious profile on lateral view (Figs. 2E, F, 3C, D, and 4C, D).

In conclusion, secondary cleft lip patients suffer from shortage of upper lip tissue, and Abbé flap can be effectively applied in these patients. A novel modification of Abbé flap can be one of the substitutes for correction for lack of central lip tubercle and deformities of Cupid's bow. Outcomes were promising, especially in aesthetic point of view.

\section{CONFLICT OF INTEREST}

No potential conflict of interest relevant to this article was reported.

\section{PATIENT CONSENT}

The patients provided written informed consent for the publication and the use of their images.

\section{REFERENCES}

1. Koshy JC, Ellsworth WA, Sharabi SE, Hatef DA, Hollier LH Jr, Stal S. Bilateral cleft lip revisions: the Abbe flap. Plast Reconstr Surg 2010;126:221-7.

2. Thomson HG, Hart NB. Reconstruction of cupid's bow: a quadrilateral flap technique. Ann Plast Surg 1989;22:195-202.

3. Abbe R. A new plastic operation for the relief of deformity due to double harelip. Plast Reconstr Surg 1968;42:481-3.

4. Peterson RA, Ellenberg AH, Carroll DB. Vermilion flap reconstruction of bilateral cleft lip deformities (a modification of the Abbe procedure). Plast Reconstr Surg 1966;38:109-15.

5. Jackson IT, Soutar DS. The sandwich Abbe flap in sceondary cleft lip deformity. Plast Reconstr Surg 1980;66:38-45.

6. Lehman JA Jr. The dynamic Abbe flap. Ann Plast Surg 1979;3:401-5.

7. Holmstrom H. The Abbe flap converted to an island flap. Scand J Plast Reconstr Surg 1986;20:51-4.

8. Whang KK, Do MO, Lee SM, Kim SH. W-modification of Abbe flap after Mohs surgery of squamous cell carcinoma on the lower lip. Dermatol Surg 2007;33:485-7.

9. McGregor IA. The abbe flap: its use in single and double lip clefts. Br J Plast Surg 1963;16:46-58.

10. Bagatin M, Most SP. The Abbe flap in secondary cleft lip repair. Arch Facial Plast Surg 2002;4:194-7.

11. Tange I. The Abbe flap for unilateral cleft lip. Ann Plast Surg 1980;5:2-6. 